\title{
Etude epidémiologique et clinique des urgences cardiovasculaires observées dans le service de cardiologie du CHU du POINT G.
}

\author{
DIALL IB1, MENTA I1, COULIBALY S1, SIDIBE N2, DIALLO BA1.
}

1. Service de cardiloogie du CHU du Point G, Bamako

2. Service de cardiologie du CHU Gabriel Touré, Bamako

\section{RESUME :}

Nous rapportont les résultats d'une étude prospective sur les urgences cardio-vasculaires réalisée sur une période d'un an dans le service de Cardiologie du CHU du Point G.

Leur prévalence était de $24,8 \%$, le sexe masculin représentait 53,7\%, l'âge moyen était de 57,42 ans; plus de la moitié des patients (64\%) étaient urbains et avaient dans $57,7 \%$ des cas un niveau de vie moyen.

Les patients ont été transférés à l'hôpital dans 93,1\% des cas, assuré par des véhicules usagers. Le délai moyen d'admission après l'apparition des premiers signes était de plus de 3 jours.

L'hypertension artérielle (HTA) avec $52 \%$ et le tabac $(37,1 \%)$ constituaient les facteurs de risque dominants.

Les principaux tableaux d'urgence étaient l'insuffisance cardiaque, l'accident vasculaire cérébral (AVC) et l'œdème aigu du poumon avec des fréquences respectives à $50,9 \%, 18,9 \%$ et $12,5 \%$. La mortalité dans la série a été de $23,4 \%$ et l'insuffisance cardiaque avec $53,8 \%$ représentait l'affection la plus meurtrière.

Mots clés : urgences, cardio-vasculaires, épidémiologie, clinique

\section{SUMMARY :}

Authors report results of a prospective study in cardiovascular emergencies in the service of cardiology $B$ at the Hospital of Point $G$ and spreaded on one year.

Their prevalence was 24,8 per cent and the man was often more touched (53,7per cent).

The average age in the sample was 57,42 ans. Patients in their majority were urban (64 per cent) and had a mean level of life $(57,7$ per cent).

The transfer to the hospital was assured in the majority of cases (93,1 per cent) by common vehicles and the average delay of admission after the apparition of first signes was more than trie days for about $2 / 3$ sick persons (61,7 per cent). The high blood hypertension (52 per cent) and the tobacco for used the dominant factors of risk.

The principal pictures of emergency were the grave heart insuffiency, the cerebral vascular accident and the occure pulmonary oedema with respective frequencies at 50,9 per cent and 12,5 per cent.

The mortality in the serries was of 23,4 per cent and the grave heart insuffiency $(53,8$ per cent) represented the most lethal affection.

Key words : emergency, cardiovascular, clinic, epidemiology

\section{INTRODUCTION}

Les pathologies cardiovasculaires qui constituent un véritable problème de santé publique dans les pays en développement se présentent très souvent sous forme d'urgence mettant en jeu le pronostic vital et / ou fonctionnel du patient et nécessitant une prise en charge précoce et appropriée. Leur prévalence et leur répartition dans l'activité quotidienne de la médecine d'urgence sont très mal connues au Mali.

En France elles représentent $15-20 \%$ des appels d'urgence du SAMU et plus de la moitié des 180000 décès annuels par pathologies cardio-vasculaires était liée aux urgences (1).

Ces pathologies constituent $5 \%$ de l'ensemble des urgences en Afrique Noire (2) et $46 \%$ des urgences médicales au Sénégal (3).

Le présent travail à pour objectif de décrire les principaux groupes nosologiques des urgences cardiovasculaires, de déterminer les affections sous-jacentes et de décrire l'évolution hospitalière.

\section{MATERIEL ET METHODE}

Nous avons réalisé une étude transversale dans le service de Cardiologie du CHU du Point G du 01/02/2012 au 29/02/2013. Ont été inclus, tout les patients hospitalisé dans le service pour urgence cardiovasculaire pendant la période d'étude.

L'urgence cardio-vasculaire était retenue devant la présence de signes physiques et lou symptômes fonctionnels mettant en jeu le pronostic vital et la nécessité d'une prise en charge rapide et appropriée du malade. Nous avons exclus de l'étude les pathologies autres qu'une cardiopathie d'urgence, et les urgences cardio-vasculaires non hospitalisées. Chaque patient a bénéficié, d'une fiche individuelle de suivi avec enregistrement systématiquement des données sociodémographiques, cliniques et de l'évolution sous traitement. La saisie des données a été faite sur le logiciel Excel-2013 et l'analyse sur SPSS 11.0 et Epi info 6 . Les tests statistiques ont été : Khi2 et le test de Fisher $p<0,05$.

\section{RESULTATS}

Pendant la période d'étude, sur 706 patients admis, 175 l'étaient pour urgence cardio-vasculaire, repartis en 94 hommes $(53,7 \%)$ et 81 femmes $(46,3 \%)$. La prévalence hospitalière des urgences cardio-vasculaires était de $24,8 \%$ et le sex-ratio de 1,1. L'âge moyen était de 57,42 ans 17,48 avec des extrêmes à 14 ans et à 90 ans et la classe modale la tranche de $60-69$ ans avec $22,9 \%$ des patients. Dans les 2 sexes, toutes les tranches d'âge 
étaient représentées avec un recrutement progressif jusqu'à 70-79 ans (tableau I).

Plus de la moitié des patients $(57,7 \%)$ avaient un niveau de vie moyen, environ $2 / 3(64 \%)$ étaient urbains.

Dans $93,1 \%$ des cas, le transfert des patients à l'hôpital était assuré par des véhicules usagers et dans 6,9\% il s'agissait d'un transfert médicalisé en ambulance. 149 malades $(85,1 \%)$ ont été admis plus de 24 heures après l'installation des premiers signes et $26(14,9 \%)$ dès le premier jour. Pour la majorité $(61,7 \%)$ le délai moyen d'admission était de plus de 3 jours. L'insuffisance cardiaque semblait être l'admission la plus tardive ; l'AVC le transfert le plus précoce et plus de la moitié des œdèmes pulmonaires arrivait à l'hôpital au-delà de 72 heures. Un peu plus du quart des patients $(26,9 \%)$ était sans antécédent et facteur de risque cardio-vasculaire. L'HTA $(52 \%)$ et le tabac $(37,1 \%)$ constituaient les facteurs de risque dominant sans cependant de corrélation statistiquement significative entre l'ancienneté de l'HTA et l'installation d'événement cardio-vasculaire urgent de même qu'entre la consommation de tabac et la survenue d'urgence cardio-vasculaire $(P \square 0,05) .102$ $(58,3 \%)$ patients avant leur accueil souffraient de pathologies cardiaques dominées par l'HTA $(41,7 \%)$ et la cardiomyopathie dilatée $(6,3 \%)$. Les principaux tableaux d'urgence étaient l'insuffisance cardiaque, l'AVC et l'œdème aigu pulmonaire avec des fréquences respectives à $50,9 \%, 18,9 \%$ et $12,5 \%$. Au total, ils représentaient plus de $3 / 4(82,3 \%)$ de l'effectif des urgences cardio-vasculaires. Une nécrose myocardique existait chez $5,1 \%$ des patients.

L'insuffisance cardiaque et la crise aiguë hypertensive prédominaient chez la femme alors que l'œdème aigu pulmonaire, le collapsus et la syncope étaient le plus souvent rencontrés chez l'homme. L'AVC touchait presque à égalité les deux sexes (tableau II).

Selon l'âge, le maximum des pathologies apparaissait dans la tranche de 60-69 ans

(73 soit $41,7 \%$ ) et seule l'insuffisance cardiaque était décrit dans les âges extrêmes (tableau III).

II n'existait pas cependant de relation statistiquement significative entre l'âge et la survenue d'événement cardio-vasculaire urgent $(P \square 0,05)$.

Le séjour hospitalier moyen était de 12,45 jours avec des extrêmes à 1 et 72 jours

L'évolution hospitalière était jugée satisfaisante chez la majorité des patients $(70,3 \%)$.Quarante et un (41) décès étaient enregistrés soit une mortalité globale de $23,4 \%$. Toutefois, il n'existait pas de différence statistiquement significative entre les létalités masculine et féminine $(P]$ 0,05).

L'insuffisance cardiaque, l'accident vasculaire cérébral et l'œdème aigu du poumon constituaient les

affections les plus meurtrières avec des létalités spécifiques respectives à $53,8 \%, 21,9 \%$ et $12,2 \%$. Ensemble elles constituaient plus de $3 / 4(87,9 \%)$ des urgences cardio-vasculaires fatales.

\section{DISCUSSION et COMMENTAIRES}

Dans l'étude, la prévalence des urgences cardiovasculaires était de $24,7 \%$ contre $17,3 \%$ chez KPÊMAHOUTOU (1). Cette prévalence est inférieure à celles de KIMOU (4) et de SYRACUSSE (5) qui respectivement étaient de $69,7 \%$ et $75,15 \%$.

Cette différence s'expliquerait par la réalisation de notre étude dans un service de Cardiologie générale et chez les précédents auteurs dans des services d'accueil d'urgence cardio-vasculaire.

L'âge moyen dans notre série était de 57,42 ans ; il était de 52 ans chez KIMOU de 50 ans chez KPÊMAHOUTOU et 48 ans chez KANE (3).

En revanche, dans les pays européens et américains, l'âge moyen des patients admis pour urgences cardiovasculaires était plus élevé : 63 ans pour SCHAMIM (6) et 67 ans dans l'étude de FRAMINGHAM (6). Ce recul de l'âge moyen par rapport à ceux des pays en développement pourrait s'expliquer par leur niveau de vie plus élevé et le meilleur accès aux soins médicaux dont ils bénéficient permettant pendant longtemps un contrôle des pathologies cardio-vasculaires

La prédominance masculine retrouvée dans l'étude est classique $(1,3,4)$ et elle est significative dans l'œdème aigu du poumon $(P=0,004)$ contrairement à l'insuffisance cardiaque où la prédominance féminine avait une significativité statistique $(\mathrm{P}=0,03)$.

L'HTA $(52 \%)$ et le tabac $(37,1 \%)$ constituaient les antécédents et facteur de risque prédominants rejoignant les observations de COULIBALY (7).

Dans la série, $12,6 \%$ des patients avaient un niveau de vie élevé en accord avec KIMOU (4) (9,2\%) et KANE (3) $(6,9 \%)$. Ce faible recrutement de patients à profil économique élevé dans l'échantillon pourrait s'expliquer par le niveau d'ensemble bas de la population malienne et aussi par l'admission assez souvent en clinique privée de patients à revenu élevé.

La majorité de nos patients (64\%) était des urbains ; observation faite également par KANE et par KPÊMAHOUTOU. Cette prédominance d'urbains, pourrait s'expliquer au Mali, en partie par le site du CHU du Point $G$ en zone urbaine et son accès difficile à nos concitoyens ruraux et démunis.

$6,9 \%$ des malades de l'étude et $5,5 \%$ des patients de KANE avaient bénéficié d'un transfert médicalisé sur l'hôpital contre $42 \%$ en France (5). Ceci traduit l'insuffisance voire l'inexistence de moyen de transfert des malades en urgence dans nos pays en développement.

Environ $2 / 3$ de nos patients (108 soit $61,7 \%$ ) consultaient dans un délai de plus de 72 heures après l'installation des premiers signes de la maladie.

A DAKAR (3) ce délai moyen était de 3 jours et au BENIN (1) d'environ 2 jours.

Dans les pays développés, il est plus court de 3 à 5 heures $(8,9)$ et témoigne alors dans nos pays de l'insuffisance de l'information et du manque de perception de l'importance de la rapidité de la prise en charge des patients. 
Plus de la moitié des patients de l'échantillon (58,3\%) souffraient de pathologies cardiaques dominées par I'HTA (41,7\%) et la cardiomyopathie dilatée (6,3\%). Cette prééminence de l'HTA et des cardiomyopathies dans les pathologies cardio-vasculaires sous-jacentes était également rapportée par KIMOU (4) alors qu'à $\operatorname{DAKAR}(3)$, I'HTA apparaissait bien loin derrière les atteintes myocardiques et les valvulopathies évoluées. Cette différence relèverait essentiellement des critères inclusifs dans les différents protocoles.

La prédominance de l'insuffisance cardiaque parmi les urgences cardio-vasculaires observées dans la série $(50,8 \%)$ est classique mais en rupture avec KIMOU (4) et BERTRAND (10), nous décrivions peu de crise aiguë hypertensive $(8 \%)$ dans la série.

Pour KANE (3) et dans l'étude SYRACUSSE (5) les troubles du rythme et de conduction représentaient les urgences cardio-vasculaires les plus courantes avec respectivement $37,4 \%$ et $25 \%$ contre $0,6 \%$ dans notre étude.

Le séjour hospitalier moyen dans la série était de 12,45 jours contre 19 heures 25 minutes à ABIDJAN où le court séjour serait le reflet de la politique en vigueur aux urgences cardiologiques et qui pourrait justifier cette différence.

Notre taux de mortalité de $23,4 \%$ était proche des $19,49 \%$ de KPEMAHOUTOU mais supérieur aux $10 \%$ en France (11) et aux 3,2\% d'ABIDJAN (4). Ce faible taux de mortalité en FRANCE et à ABIDJAN est au moins en partie lié à l'existence d'unités de soins intensifs cardiologiques.

A DAKAR, pour KANE (3) l'évolution est fatale chez la moitié des malades.

Le pronostic dans l'ensemble effroyable dans nos pays à revenu faible est plurifactoriel : possibilités diagnostiques limitées, inaccessibilité de certaines thérapeutiques d'urgence et accueil des malades dans un tableau avancé de déchéance myocardique.

L'insuffisance cardiaque était l'affection la plus meurtrière avec plus de la moitié des décès $(53,6 \%)$; à ABIDJAN (4) elle était également comptable de la majorité des décès mais au BENIN, la crise hypertensive avec $23,8 \%$ était la pathologie la plus létale contre $2,4 \%$ dans notre série et $7,7 \%$ chez SARR et coll (12).

\section{CONCLUSION}

Les urgences cardio-vasculaires constituent un réel et majeur problème de santé au MALI par leur fréquence et leur gravité évolutive en l'absence de prise en charge adéquate et rapide.

Aussi la réduction de leur morbimortalité passe par l'amélioration du plateau technique pour une approche diagnostique et thérapeutique efficiente.,l'ouverture d'une unité de soins intensifs cardiologiques et l'information et l'éducation des populations et notamment des groupes cibles sur les risques cardio-vasculaires.

\section{REFERENCES}

1- AGBOTON H.Les urgences cardiovasculaires au Bénin: problèmes courants attitude pratique. RAMUR $2000 ; 5(2)$ : 83-84.

2- KPÊMAHOUTON R. Panorama et prise en charge des urgences cardiovasculaires à l'USIC de l'USERC du CNHU H.K.M de COTONOU.Thèse, Médecine, Cotonou $2002 ; 139$.

3- A KANE, A.L.S N'DIAYE, M DIAO, G KIMBALLYKAKI, I.B DIOP, M SAAR, S.A BA, S.M DIOUF.

Prise en charge des urgences cardiovasculaires au Sénégal. Card. Tropicale 2002 ; 28 (109) : 15-16.

4- KIMOU A.

Les urgences cardiovasculaires à l'Institut de Cardiologie d'ABIDJAN. Thèse, Médecine, Abidjan 2004 ; 117.

\section{5-JACOB D.}

Étude SYRACUSE : 24 heures d'urgences cardiovasculaires.La revue du Cardiologue praticien $1996 ; 117$.

6- KOEGLER A, ROUL G, BAREISIS, RIESP $G$ et al. Mortalité à 4 ans de l'insuffisance cardiaque à fonction systolique conservée.

Arch. Mal. Cœur 1995 ; 88 : 961-966.

7- COULIBALY T.

Étude des accidents vasculaires cérébraux du sujet jeune dans les services de cardiologie et de neurologie au Mali. Thèse, Médecine, Bamako 2001 ; 132.

8- CASTILLO F.A, MEDVEDOWSKY J.L,ABASTADO M.Enquêté nationale sur les urgences cardio vasculaires : modalité d'admission en unité de soins intensifs cardiologiques. Archive maladie du cœur et des vaisseaux : $1987 ; 1179-88$.

9- THE EUROPEAN MYOCARDIAL INFARCTION GROUP. Prehospital thrombolytic therapy in patients with suspected acute myocardial infarction.N Engl Med : $1993 ; 329: 383-9$.

10- BERTRAND Ed et al.Observation sur les cardiopathies des Africains à DAKAR BULL Médecine, A.O.F, 1951; 8.

11- Recommandations de la société française de Cardiologie pour la prise en charge des urgences cardiologiques. Arch. Mal cœur : 1999 ; 92 : 337-45.

12- SARR M, DIAOM, THIAM et al. Les urgences hypertensives en milieu cardiologique à DAKAR à propos de 65 cas. RMUR 2000 ; 5 (2) : 92-93. 
Tableau I: répartition des patients en fonction de l'âge et du sexe.

\begin{tabular}{lccc} 
& \multicolumn{2}{c}{ Sexe } & Total \\
\cline { 2 - 3 } Age ans) & Féminin & Masculin & \\
\hline $10-19$ & 5 & 1 & 6 \\
$20-29$ & 7 & 2 & 9 \\
$30-39$ & 9 & 4 & 13 \\
$40-49$ & 8 & 13 & 21 \\
$59-59$ & 14 & 19 & 33 \\
$60-69$ & 15 & 25 & 40 \\
$70-79$ & 15 & 23 & 38 \\
$80-89$ & 7 & 7 & 14 \\
$90-99$ & 1 & 0 & 1 \\
\hline Total & 81 & 94 & 175 \\
\hline
\end{tabular}

Tableau II : répartition des patients en fonction du sexe et de l'urgence cardio-vasculaire

\begin{tabular}{llll}
\hline Urgencecardio-vasculaire(UCV) & Féminin & Masculin & Total \\
\hline InsuffisancecardiaquestadeIV(IC) & $48(27,4 \%)$ & $41(23,4 \%)$ & $89(50,8 \%)$ \\
Accidentvasculairecérébral(AVC) & $17(09,7 \%)$ & $16(09,1 \%)$ & $33(18,8 \%)$ \\
CEdèmeaigupulmonaire(OAP) & $04(02,2 \%)$ & $18(10,3 \%)$ & $22(12,5 \%)$ \\
Lacriseaiguëhypertensive(HTA) & $09(05,1 \%)$ & $05(02,9 \%)$ & $14(08,0 \%)$ \\
Infarctusdumyocarde(IDM) & $02(01,1 \%)$ & $07(04,0 \%)$ & $09(05,1 \%)$ \\
Collapsusouchoc & $00(00,0 \%)$ & $04(02,3 \%)$ & $04(02,3 \%)$ \\
Syncope & $00(00,0 \%)$ & $02(01,1 \%)$ & $02(01,1 \%)$ \\
Emboliepulmonaire(EP) & $01(00,6 \%)$ & $00(00,0 \%)$ & $01(00,6 \%)$ \\
Troubledurythme(TR) & $00(00,0 \%)$ & $01(00,6 \%)$ & $01(00,6 \%)$ \\
\hline Total & $81(46,1 \%)$ & $94(53,9 \%)$ & $175(100 \%)$ \\
\hline
\end{tabular}

Tableau III : Répartition des patients en fonction de l'âge et de l'urgence cardio-vasculaire

\begin{tabular}{lllllllllll} 
& \multicolumn{1}{c}{ Age en année } & \multicolumn{1}{c}{ Total } \\
\cline { 2 - 9 } UCV & $10-19$ & $20-29$ & $30-39$ & $40-49$ & $50-59$ & $60-69$ & $70-79$ & $80-89$ & $90-99$ & \\
IC & 4 & 7 & 10 & 11 & 12 & 15 & 19 & 9 & 1 & 89 \\
AVC & 1 & 1 & 0 & 4 & 6 & 11 & 6 & 4 & 0 & 33 \\
OAP & 0 & 0 & 2 & 3 & 3 & 7 & 6 & 1 & 0 & 22 \\
HTA & 0 & 0 & 1 & 2 & 4 & 2 & 5 & 0 & 0 & 14 \\
IDM & 0 & 0 & 0 & 1 & 5 & 3 & 0 & 0 & 0 & 9 \\
Collapsus & 1 & 0 & 0 & 0 & 1 & 2 & 0 & 0 & 0 & 4 \\
Syncope & 0 & 0 & 0 & 0 & 1 & 0 & 1 & 0 & 0 & 2 \\
EP & 0 & 0 & 0 & 0 & 0 & 0 & 1 & 0 & 0 & 1 \\
TR & 0 & 0 & 0 & 0 & 1 & 0 & 0 & 0 & 0 & 1 \\
\hline Total & 6 & 8 & 13 & 21 & 33 & 40 & 38 & 14 & 1 & 175 \\
\hline
\end{tabular}




\title{
Améliorer les pratiques et l'organisation des soins : méthodologie des revues suystématiques
}

\author{
Improving practice and organisation of care: methodology for systematic reviews.
}

\author{
(In Santé Publique, Volume 26/№5 - septembre-Octobre 2014 pp: (655-667)) \\ Vincent Zaugg ${ }^{1}$, Virginie Savoldelli ${ }^{1,2}$, Brigitte Sabatier ${ }^{1,3}$, Pierre Durieux ${ }^{1,3}$ \\ 1 Hôpital Européen Georges Pompidoiu, 20 rue Leblanc, 75015 Paris \\ 2 Université Paris Sud - Faculté d Pharmacie, Département de pharmacie clinique, EA123, barrières physiologiques et réponses thérapeutiques \\ 3 INSERM , Institut des cordeliers, UMRS 1138 eq22, centre Cohrance Français.
}

\begin{abstract}
Résumé
Le nombre d'études d'interventions visant à améliorer la qualité des soins est en constante augmentation, si bien qu'il est difficile d'accéder à toute l'information disponible sur un sujet La revue systématique est un outil permettant de fournir rapidement une information exhaustive et objective aux professionnels de la santé. Cet article a pour objectif de détailler les étapes de la réalisation d'une revue systématique: définition de la question de recherche, recherche et sélection des études, extraction et analyse des données, évaluation de la qualité méthodologique des études, synthèse des résultats. Les interventions destinées à améliorer les pratiques et l'organisation des soins présentent certaines spécificités qui conditionnent la méthodologie de la revue systématique. II faut par exemple prendre en compte la variabilité importante qui existe souvent entre les populations, les organisations et les interventions d'une étude à l'autre, ce qui rend difficile la synthèse des résultats sous forme de métaanalyse. La connaissance des particularités des revues systématiques d'interventions est indispensable, que ce soit pour réaliser une synthèse de la littérature de qualité, ou pour évaluer le niveau de preuve d'une revue systématique publiée.
\end{abstract}

Mots-clés: Études d'intervention; Qualité des soins de santé; pratique professionnelle; Évaluation des résultats et des processus en soins de santé; Organisation des soins; Revue de la littérature.

\begin{abstract}
Summary
The number ofintervention studies designed to improve quality of care is increasing exponentially, making it difficult to access ail available information on a given subject. Systematic reviews are tools that provide health professionals with comprehensive and objective information. This article describes the main phases of a systematic review: formulating the research question, search and selection of studies, data extraction and analysis, assessment of the methodological quality of studies, and synthesis of the results. Interventions designed to improve professional practices and orqanisation of care have specifie characteristics that determine the methodology of systematic reviews. For example, the often substantial heterogeneity between populations, organisations, and intervention settings among studies must be taken into account; which makes meta-analysis more difficult. Knowledge on specifie features of system a tic reviews designed to improve quality of care is essential to ensure a good review of the literature, or to evaluate the level of evidence of published systematic reviews.
\end{abstract}

Keywords: Intervention Studies; Quality of Health Care;
Professional Practice; Outcome and Process Assessment Health Carel; Organisation of Care; Review.

\section{Introduction}

Une revue systématique consiste en une synthèse de la littérature scientifique en réponse à une question précise. Elle utilise des méthodes explicites de recherche, de sélection et d'analyse des données. Des méthodes statistiques peuvent ou non être utilisées pour synthétiser les résultats des études. Lorsque de telles méthodes sont employées, on parle de méta-analyse [1]. Une revue systématique est donc une vraie méthode de recherche, etne doitpas être confondue avec une revue générale dans laquelle la recherche bibliographique n'est en général pas exhaustive et qui représente plus l'opinion d'un expert ou d'un groupe d'experts. Les revues systématiques permettent aux professionnels de la santé d'accéder à une information valide et contribuent ainsi à orienter les décisions. Les lecteurs doivent en connaître les règles méthodologiques et être en mesure d'en évaluer la qualité. Les revues systématiques se sont considérablement développées ces dernières années pour évaluer les stratégies thérapeutiques et se sont étendues plus récemment à d'autres domaines, diagnostique et pronostique par exemple. Dans le champ de la qualité et de l'organisation des soins, où le nombre d'études d'intervention publiées est en constante augmentation, les revues systématiques ont également leur place. Cet article a pour objectif de décrire les étapes et les spécificités méthodologiques d'une revue systématique d'études d'interventions, en se fondant notamment sur les recommandations du groupe EPOC [2] (Effective Practice and Organisation of Care) de la collaboration Cochrane. Ce groupe publie des revues systématiques sur les interventions destinées " à promouvoir la pratique professionnelle la plus efficace ", qu'il s'agisse d'interventions sur les professionnels de santé ou sur le financement ou l'organisation des soins.

\section{Rédaction et enregistrement du protocole}

La réalisation d'une revue systématique constitue un travail complexe qui répond aux mêmes règles que toute étude de recherche. Sa première étape est donc la conception et la rédaction d'un protocole. Celui-ci permet de limiter les biais et d'assurer la meilleure objectivité à la revue systématique. Pour sa rédaction, les auteurs peuvent s'appuyer sur les 